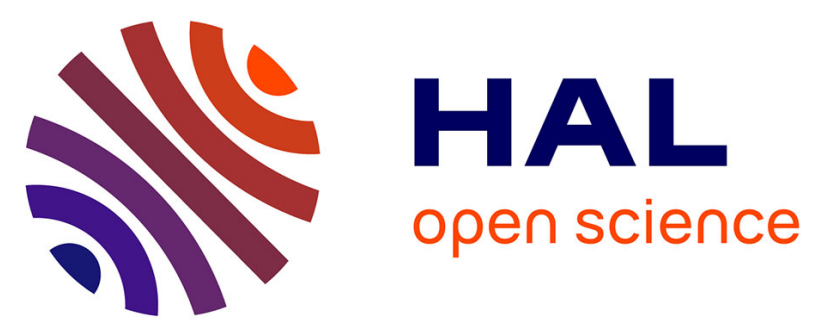

\title{
A Numerically Efficient Method for Predicting the Scattering Characteristics of a Complex Metallic Target Located Inside a Large Forested Area
}

\author{
Lydia Hettak, Hélène Roussel, Massimiliano Casaletti, Raj Mittra
}

\section{To cite this version:}

Lydia Hettak, Hélène Roussel, Massimiliano Casaletti, Raj Mittra. A Numerically Efficient Method for Predicting the Scattering Characteristics of a Complex Metallic Target Located Inside a Large Forested Area. IEEE Transactions on Geoscience and Remote Sensing, 2017, PP (99), pp.1 - 9. 10.1109/TGRS.2017.2761023 . hal-01656852

\section{HAL Id: hal-01656852 \\ https: / hal.sorbonne-universite.fr/hal-01656852}

Submitted on 5 Mar 2018

HAL is a multi-disciplinary open access archive for the deposit and dissemination of scientific research documents, whether they are published or not. The documents may come from teaching and research institutions in France or abroad, or from public or private research centers.
L'archive ouverte pluridisciplinaire HAL, est destinée au dépôt et à la diffusion de documents scientifiques de niveau recherche, publiés ou non, émanant des établissements d'enseignement et de recherche français ou étrangers, des laboratoires publics ou privés. 


\title{
A Numerically Efficient Method for Predicting the Scattering Characteristics of a Complex Metallic Target Located Inside a Large Forested Area
}

\author{
L. Hettak Student Member, IEEE, H. Roussel Member, IEEE, M. Casaletti Member, IEEE, R.Mittra \\ Life Fellow, IEEE
}

\begin{abstract}
An efficient electromagnetic model for the scattering analysis of targets placed in large natural environments is presented. A hybrid formulation combining volume and surface integral equations is used to describe forest environment (dielectric objects) in which metallic structures are present. A large part of the forest can be analyzed electromagnetically by using the Characteristic Basis Function Method (CBFM) whose use enables us to simulate the problem at hand and significantly reduce the dimension of the linear system that needs to be solved.
\end{abstract}

Index Terms - CBFM, hybrid formulation, Foilage Penetrating (Fopen)

\section{INTRODUCTION}

$\mathrm{R}$ ecent years have witnesed a great deal of interest in the developement of Foliage Penetrating (FoPen) radars since they have a wide range of civilian and military applications. Since low frequency electromagnetic waves penetrate through forest foliage, these frequency bands (VHF, UHF, L and S) are typically used for this type of applications [1]-[6]. However, we note that FoPen radar development is very expensive and requires extensive measurement programs. The development of a "full wave" electromagnetic model for analyzing scattering from large forest areas surrounding metallic targets can significantly reduce the number of such measurements.

Indeed, the use of such models could substitute the first steps of radar developement by identifiying the types of radar configurations which should be investigated.

The development of exact electromagnetic models of forest scenarios presents two main challenges: (i) First, the scene is composed of highly heterogeneous media (lossy

Manuscript received April 19,2017

L. Hettak, H. Roussel and M. Casaletti are with the Sorbonne Universités, UPMC Univ. Paris 06, UR2, L2E, F-75005, Paris, France (e-mail: lydia.hettak, helene.roussel, massimiliano.casaletti@upmc.fr).

R. Mittra / EMC Lab , University of Central Florida, Orlando,

FL USA and King Abdul Aziz University, Jeddah, Saudi Arabia. (e-mail: rajmittra@ieee.org). dielectric materials and metals); (ii) The size of the illuminated area (the focal spot of the UHF radar antenna) could be up to $50 \times 50 \mathrm{~m}^{2}$ which corresponds to $65 \times 65$ wavelentgh at $400 \mathrm{MHz}$.

Several techniques have been proposed in the literature for electromagnetic simulation of $50 \times 50 \mathrm{~m}^{2}$ this type of scenarios. In [4], a hybrid FDTD formulation using Huygens' surface approach for the metallic target and a coherent single scattering theory for the forest has been proposed. This model accounts for the coupling between the target and the foilage by using an iterative physical optics (PO) approach [5]. In [6], a Multilevel Fast Multipole Algorithm (MLFMA) has been used to evaluate the scattering characteristics of electrically large conducting and dielectric targets placed at the interface of a dielectric half-space below and air above.

Several full-wave techniques can be used to evaluate the field scattered by a heterogeneous environment (dielectric and metallic media). A surface integral formulation has been employed in [7] for both metallic and dielectric scatterers. However, this method requires the decomposition of the scene into several homogeneous dielectric sub problems, which is not possible for a natural environment. A second approach is to combine a volume integral formulation for dielectrilc objects with a surface formulation for the metallic ones [8], and we opt to use the latter in this work because of its generality. This choice is also justified by the fact that the volume formulation of the electric field integral equation has been successfully used by our group to model the "forest only" scene.

In this paper, we present a new approach for detecting targets camouflaged in a forest. This model is based on a hybrid surface/volume integral formulation which uses the Characteristic Basis Function Method (CBFM) applied in the context of the VEFIE [9]-[11] to construct a numerical Green's function of the natural environment.

The scattered electromagnetic field is obtained by solving an Electric Field Integral Equation (EFIE) for the metallic objects using a Method of the Moment (MoM) scheme [12][15]. In addition to detecting targets under cover, the proposed 
model can also treat problems related to the forest, such as the study of the biomass and the influence of moisture on the diffracted field. The target is supposed to be a Perfect Electric Conductor (PEC), while the ground is assumed to be flat. This latter limitation can be overcome by modeling the ground deformations as dielectric objects with the same electromagnetic characteristic as those of the ground.

The paper is structured as follows. Section II is a step by step description of the formulation of our model named DEMOS (Domain dEcomposition MOdel for the analysis of target Scattering in forested areas). Section III presents a validation of the proposed method through comparison with the results derived by using the commercial software (FEKO). Section IV presents the DEMOS analysis of a realistic scenario in order to highlight the effect of camouflaged target on the fields scattered by a forest area. Finally, conclusions are drawn in section $\mathrm{V}$.

\section{FORMULATION}

A typical scene of interest for our investigation is described in Fig.1. A metallic object is placed in a locally flat natural environment and surrounded by trees. From an electromagnetic point of view, the scene is composed of two classes of elements: the dielectric forest and the metallic target.

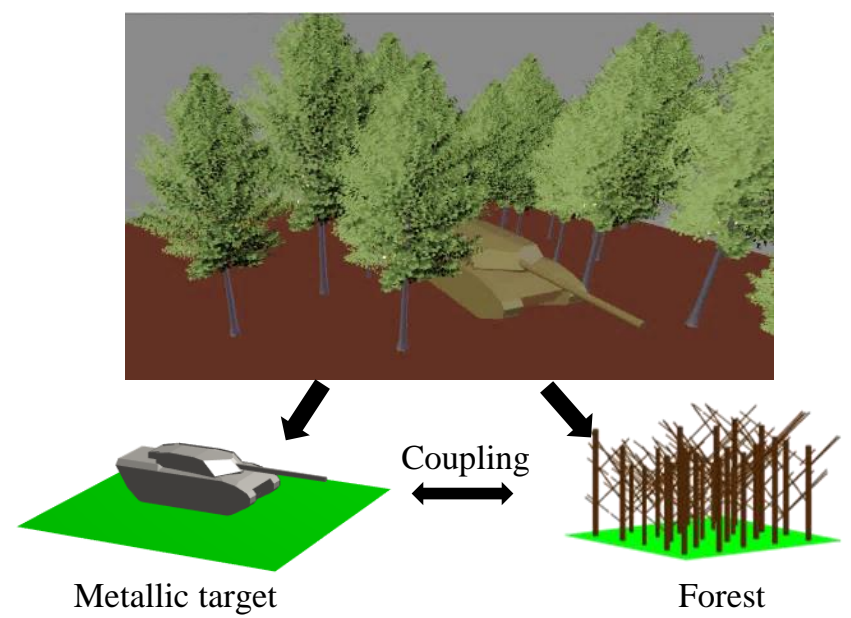

Fig.1. Example of representation of a typical scene

In this section, we introduce the formulations for analysing each of the two elements of the scene. We will begin by modeling the problem of scattering by a large forest area. Next, the model used to calculate the scattering of a metallic target placed above a lossy ground is developed. Finally, the two models are combined by using a hybrid formulation in order to include the coupling between the two elements in the model.

Throughout this paper, vectors will be denoted by bold characters and tensors by bold characters underlined by a double bar. An $e^{+j \omega t}$ time dependence is assumed. The freespace propagation constant $k_{0}$ is defined as $k_{0}=\omega \sqrt{\varepsilon_{0} \mu_{0}}=2 \pi / \lambda_{0}$ where $\mu_{0}, \varepsilon_{0}$ and $\lambda_{0}$ are respectively the permeability, permittivity and the wavelength in free space. The observation and source point are represented respectively by $\mathbf{r}$ and $\mathbf{r}^{\prime}$.

A. 3D-Full wave electromagnetic model of the large forest area

In the frequency range between 100 to $400 \mathrm{MHz}$ (UHFVHF) the main scatterers are the tree trunks and the primary branches [16]. As a result, the forest can be represented by vertical and titled dielectric cylinders as shown in Fig.2.

In each point of the scene $\mathbf{r}$ the total electric field $\mathbf{E}$ has to satisfy the Volume Electric Field Integral Equation (VEFIE) [9] given by

$$
\mathbf{E}(\mathbf{r})=\mathbf{E}_{i n c}(\mathbf{r})+\left[\nabla \nabla+k_{0}^{2}\right] \iiint_{\Omega} \Delta \varepsilon\left(\mathbf{r}^{\prime}\right) \underline{\underline{\mathbf{G}}}\left(\mathbf{r}, \mathbf{r}^{\prime}\right) \mathbf{E}\left(\mathbf{r}^{\prime}\right) d \mathbf{r}^{\prime}
$$

where $\mathbf{E}_{\text {inc }}$ is the incident electric field, $\Delta \varepsilon\left(\mathbf{r}^{\prime}\right)=\left(\varepsilon\left(\mathbf{r}^{\prime}\right)-\varepsilon_{0}\right) / \varepsilon_{0}$ is the dielectric contrast at a source

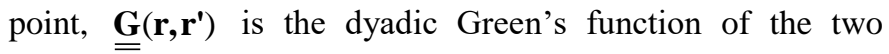
layered media (free-space and the ground), and $\Omega$ is the domain occupied by the scatterers (trunks and main branches).

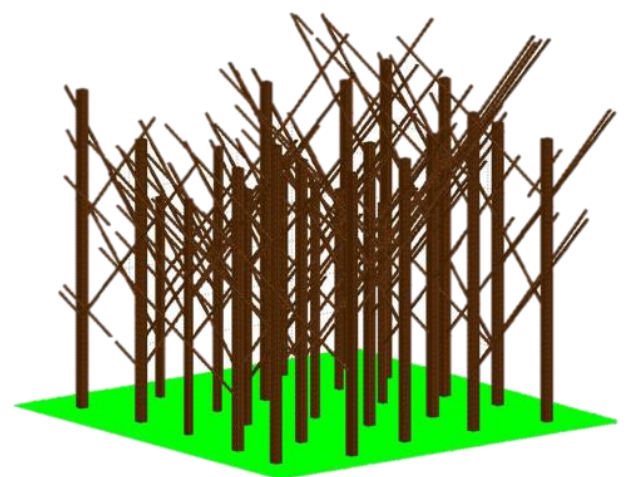

Fig. 2. Example of representation of a typical forested area. The trees are described by vertical cylinders (trunks) and tilted cylinders (primary branches) at VHF frequencies (around $400 \mathrm{MHz}$ ).

Equation (1) is solved using the Method of Moments (MoM) which is used to convert it into a matrix equation. The dielectric cylinders are discretized into $\mathrm{N}$ cubic cells of dimension smaller or equal to $\lambda_{s} / 10$ (where $\lambda_{s}$ is the wavelength inside the scatterers) so that the field inside each cell can be considered as constant. Hence, uniform basis functions and Dirac test functions (point matching method) have been used. In order to consider very large scenes and overcome the limitations introduced by the large number of unknowns, an Extended-CBFM (CBFM-E) compression scheme has been implemented [10]. The algorithm begins with a domain decomposition of the scene into several geometrical smaller blocks. In each block, Characteristic Basis Functions (CBFs) are generated by aggregating uniform (low-level) basis functions. The number of CBFs retained for each block is much lower than the original number of low-level basis functions, leading to a significant MoM interaction matrix compression.

This scheme can easily handle problems involving millions of unknowns with a significant reduction of computational time and memory requirement with respect to conventional 
MoM approach.

\section{B. Model of the diffraction of the metallic targets}

In this section we analyze the diffraction phenomenon associated with arbitrary shaped metallic structures placed over a dielectric ground.

The electric field scattered by a metallic object can be expressed as

$$
\mathbf{E}^{s, m}(\mathbf{r})=-j \omega \mu_{0} \int_{S} \underline{\underline{\mathbf{G}}}\left(\mathbf{r}, \mathbf{r}^{\prime}\right) \mathbf{J}_{\mathbf{s}}\left(\mathbf{r}^{\prime}\right) \mathbf{d} \mathbf{r}^{\prime}
$$

where $\mathbf{J}_{\mathbf{s}}\left(\mathbf{r}^{\prime}\right)$ is the unknown electric current induced on the conducting surface $S$.

The total tangent field has to vanishing on the PEC objects surfaces, leading to the Electric Field Integral Equation (EFIE):

$$
-\hat{\mathbf{n}} \times \mathbf{E}^{i n c}(\mathbf{r})=\hat{\mathbf{n}} \times\left(-j \omega \mu_{0} \int_{S} \underline{\underline{\mathbf{G}}}\left(\mathbf{r}, \mathbf{r}^{\prime}\right) \mathbf{J}_{\mathbf{s}}\left(\mathbf{r}^{\prime}\right) \mathbf{d} \mathbf{r}^{\prime}\right)
$$

where $\hat{\mathbf{n}}$ is the normal unit vector of the surface.

The MoM is used to numerically solve (3) by discretizing $\mathrm{S}$ with a triangular mesh and using the Rao-Wilton-Glisson (RWG) basis and test functions [12].

\section{Approximation of the Green's function of a two layer medium}

In the models presented heretofore, the metallic target and the trees are lying on a horizontal plane separating two semiinfinite homogeneous mediums (the ground and free space). The soil roughness is assumed to be negligible in the considered frequency band. The Green's function calculation of the vector and scalar potentials for a layered medium requires the evaluations of several Sommerfeld-type integrals [13]-[15]. To avoid doing these calculations, and thereby speeding up our computation significantly, a complex image approximation based on Fresnel reflection coefficients is introduced.

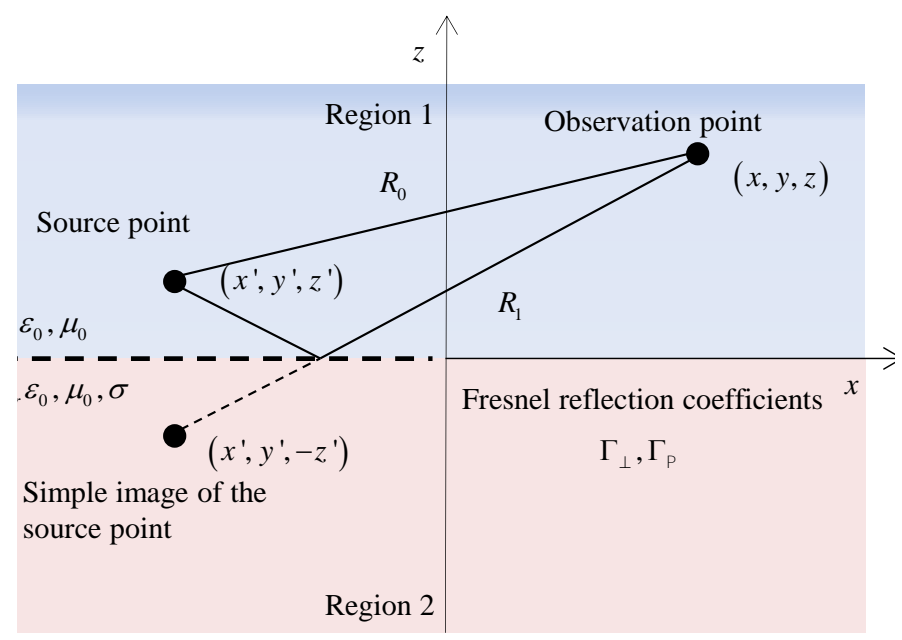

Fig. 3. Image principle geometry using Fresnel reflection coefficients.

In this approach, an image source is placed in the specular position with respect to the plane as for the PEC case, while its amplitude is modified by the Fresnel reflections coefficients given by:

$$
\left\{\begin{array}{l}
\Gamma_{\perp}=\frac{k_{z 0}-k_{z 1}}{k_{z 0}+k_{z 1}} \\
\Gamma=\frac{n_{1} k_{z 0}-k_{z 1}}{n_{1} k_{z 0}+k_{z 1}}
\end{array}\right.
$$

where $k_{z i}=\sqrt{k_{i}^{2}-k_{\rho}^{2}}$ (with $\mathrm{i}=0,1$ ) are the vertical wave numbers in free space and in the ground medium, $k_{\rho}$ is the radial wave number, and $n_{1}$ is the refractive index of the lossy ground.

The field $\mathbf{E}_{i}$ radiated by the image source is thus expressed as

$$
\mathbf{E}_{i}(\mathbf{r})=\Gamma_{\perp} \mathbf{E}_{p}(\mathbf{r})+\left(\Gamma-\Gamma_{\perp}\right)\left(\mathbf{E}_{p}(\mathbf{r}) \cdot \hat{\mathbf{u}}\right) \cdot \hat{\mathbf{u}}
$$

where $\mathbf{E}_{p}$ is the field radiated by the a source placed at the image position and $\hat{\mathbf{u}}=\left(\mathbf{r}-\mathbf{r}_{\text {image }}^{\prime}\right) \times \hat{\mathbf{z}}$.

Note that the reflection coefficients for a perfectly conducting earth are $\Gamma_{\perp}=-1, \Gamma=1$.

At the end, an approximated equivalent problem is obtained where source and its image radiate in free space (figure 3).

\section{DEMOS : Domain dEcomposition MOdel for Scattering in forest environments}

The two previous formulations are now combined in order to account for the coupling between the metallic target and the dielectric forest. The model used for metallic objects requires a surface discretization while the one for the forest needs a volume discretization. It is therefore necessary to implement a hybrid formulation to combine the two methods.

The fields scattered by both type of objects (metallic and dielectric) has to verify equations (1) and (3), leading to the following coupled equations

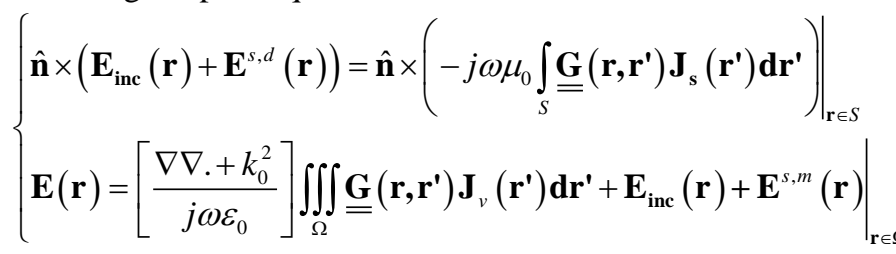

where $\mathbf{E}^{s, d}$ is the field scattered by the forest in the presence of the target.

A MoM approach is used to solve (6).by projecting (testing) each equation on an adequate basis function set. RWG basis functions for the surface equation (metallic object) and the parallelepiped constant functions for the volume one (dielectric forest). Analogously, the unknown currents densities induced on the surface of the metal and volume of the dielectric are expressed as:

$$
\left\{\begin{array}{l}
\mathbf{J}_{\mathbf{s}}=\sum_{n=1}^{N_{s}} \mathbf{I}_{\mathbf{s}} \mathbf{f}_{\mathbf{n}}^{\mathbf{R W G}} \\
\mathbf{J}_{\mathbf{v}}=j \omega\left(\varepsilon\left(\mathbf{r}^{\prime}\right)-\varepsilon_{0}\right) \mathbf{E}\left(\mathbf{r}^{\prime}\right)=\sum_{n=1}^{N_{v}} \mathbf{I}_{\mathbf{v}} \mathbf{f}_{\mathbf{n}}^{\text {rect }}
\end{array}\right.
$$

leading to the following block matrix system: 


$$
\left[\begin{array}{ll}
\underline{\underline{\mathbf{Z}}}_{s s} & \underline{\underline{\mathbf{Z}}}_{s v}^{\mathbf{Z}} \\
\underline{\underline{\mathbf{Z}}}_{v s} & \underline{\underline{v}}_{v v}
\end{array}\right]\left[\begin{array}{l}
\mathbf{I}_{s} \\
\mathbf{I}_{v}
\end{array}\right]=\left[\begin{array}{l}
\mathbf{V}_{s} \\
\mathbf{V}_{v}
\end{array}\right]
$$

where $\underline{\underline{\mathbf{Z}}}_{s s}, \underline{\underline{\mathbf{Z}}}_{v v}$ are the self-coupling matrices of the metallic and dielectric structures, and $\underline{\underline{\mathbf{Z}}}_{v s}, \underline{\underline{\mathbf{Z}}}_{s v}$ represent the coupling matrices between the metallic structure and the forest. Also, $\mathbf{I}_{s}$ and $\mathbf{I}_{v}$ are the unknown surface and volume current amplitudes, while $\mathbf{V}_{s}$ and $\mathbf{V}_{v}$ are the source vectors.

We rewrite the linear system (8) by solving separately the two unknown currents terms ( $\mathbf{I}_{s}$ and $\mathbf{I}_{v}$ ):

$$
\begin{aligned}
& {\left[\underline{\underline{\mathbf{Z}}}_{s s}-\underline{\underline{\mathbf{Z}}}_{\mathrm{c}, s}\right] \mathbf{I}_{s}=\mathbf{V}_{s}-\underline{\underline{\mathbf{Z}}}_{s v} \underline{\mathbf{Z}}_{v v}^{-1} \mathbf{V}_{v}}
\end{aligned}
$$

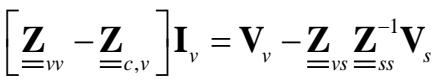

where $\underline{\underline{\mathbf{Z}}}_{c, s}=\underline{\underline{\mathbf{Z}}}_{s v} \underline{\underline{\mathbf{Z}}}_{v v}^{-1} \underline{\mathbf{Z}}_{v s}$ and $\underline{\underline{\mathbf{Z}}}_{c, v}=\underline{\underline{\mathbf{Z}}}_{v s} \underline{\mathbf{Z}}_{s s}^{-1} \underline{\mathbf{Z}}_{s v}$ represent the coupling matrices.

Equation (9) can be interpreted as a EFIE scheme for the surface currents using the VEFIE formulation as numerical Green's function. System (10), represents a VEFIE scheme for the volume currents using the EFIE formumation as numerical Green's function.

Matrices representing volume current interactions are compressed by the CBFM_E approach enabling us to handle very large scenarios. Consequently, our model (DEMOS) can treat realistic forest areas and their interaction with large metallic targets.

\section{NUMERICAL RESULTS}

This section presents a numerical validation of the proposed model (DEMOS) via a comparison with the commercial software FEKO. Each step of our formulation is validated separately.

\section{A. Dipole source above a lossy ground}

In order to investigate the accuracy of the approximated approach based on the use of the Fresnel reflections coefficients, we consider an arbitrary electric dipole placed in the free-space (region-1) above a lossy ground (region-2), as illustrated in Fig.3. Since an arbitrary current distribution can be decomposed as a sum of vertical and horizontal elementary dipoles, the accuracy of the radiation characteristics for these cases have been investigated. A lossy ground of relative permittivity $\varepsilon_{r}=7$, and conductivity $\sigma=0.02 \mathrm{~S} / \mathrm{m}$ has been considered. The source point is located at $\left(x^{\prime}, y^{\prime}, z^{\prime}\right)=(0,0,0.1) \mathrm{m}$, and the working frequency is $f=500 \mathrm{MHz}$.

The field diffracted by the source dipole has been calculated by using three different methods. The first one is the rigorous Sommerfeld calculation carried out by using FEKO. The second method is based on a two level discrete complex image method [16]-[18], while the third one is the proposed method using Fresnel reflections coefficients.

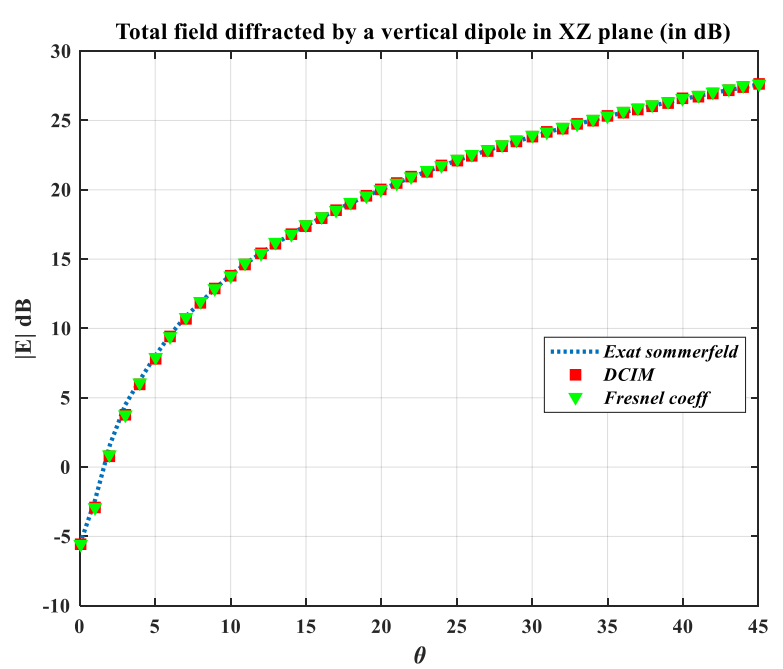

(a)

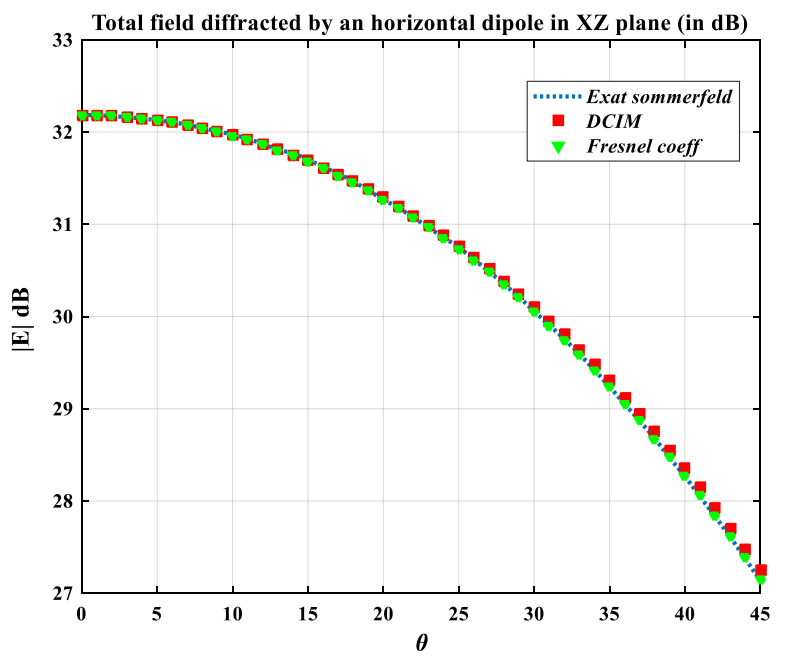

(b)

Fig.4. Comparison of the amplitude of the total field generated by a dipole at $r=5 \mathrm{~m}$ : (a) vertical dipole (b) horizontal dipole.

Figure 4 presents a comparison of the amplitude of the diffracted field (polar $\mathrm{HH}$ ) obtained by using the three methods. We observe that good agreement is obtained between our model and the two other methods. The use of the Sommerfeld integrals provides the rigorous solution. However, we observe that the use of the Fresnel reflection coefficients provides an accurate result demanding a significantly lower execution time. Such reduction in CPU time is of great interest when dealing with large scenes.

\section{B. Scattering by the trees}

In Section II, we defined a model to evaluate the field scattered by the forest. This model has already been presented in previous research works of our group [9]-[10] and has been proven to be efficient. In this paper, we analyze the influence of the permittivity of the trees (affected by humidity) on the scattered field. The test configuration is composed by two identical trees spaced $2 \mathrm{~m}$ apart along the OX-axis. The geometrical model of the trees was created by using the opensource random tree generator Arbaro [20]. The tree mesh was then exported from Arbaro to our model. In the VHF-UHF 
bands, the corresponding equivalent model is composed of only the trunk and primary branches as they are the only parts that interact with the electromagnetic wave. Quaking aspen trees have been chosen for our test (Fig. 5).

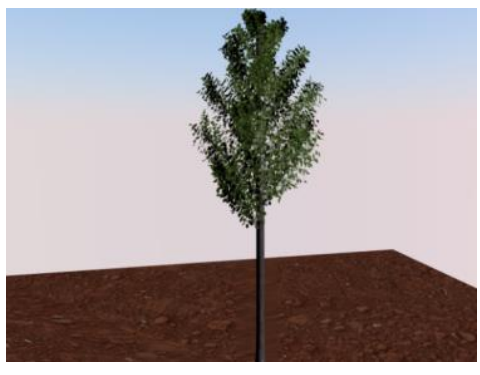

(a)

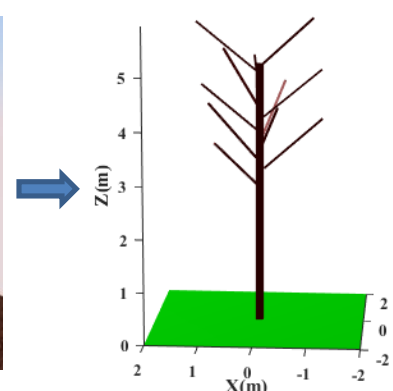

(b)
Fig. 5. Quacking aspen tree. (a) model generated by Arbaro [20]. (b) equivalent model in the VHF- UHF bands

The trees are composed of a trunk of $5 \mathrm{~m}$ height, a base diameter of $30 \mathrm{~cm}$ and 11 primary branches. The trees and the ground are assumed to be electrically homogeneous. The relative dielectric constant $\varepsilon_{r}$ and conductivity $\sigma$ of the ground are 5.45 and $20 \mathrm{mS} / \mathrm{m}$, respectively. Dry (low-loss) and wet (lossy) trees cases are investigated. For both cases, the material parameters $\left(\varepsilon_{r}\right.$ and $\left.\sigma\right)$ are equal to (4.2 and $10^{-4} \mathrm{~S} / \mathrm{m}$ ) for the low-loss case of dry trees, and (13.9 and $39 \mathrm{mS} / \mathrm{m}$ ) for the lossy case of wet trees, respectively [21].

The incident field is a plane wave propagating along the direction defined by $0^{\circ} \leq \theta_{\text {inc }} \leq 90^{\circ}$ and $\phi_{\text {inc }}=0^{\circ}$. The considered frequency is $f=400 \mathrm{MHz}$ and the number of unknowns is $\mathrm{N}=7062$ for the case of dry trees, while it is $\mathrm{N}=12978$ for the wet trees. In Fig.7 and Fig.8, we compare the $(V V)$ and $(H H)$ monostatic RCS results (with respect to the incident angle $\theta$ ) obtained by using our model and FEKO. We note that a good agreement is obtained even for the lossy trees. Furthermore, the influence of the humidity of the trees on the RCS is remarkable. Specifically, the fields diffracted by the wet trees for both polarizations are greater than dry trees case.

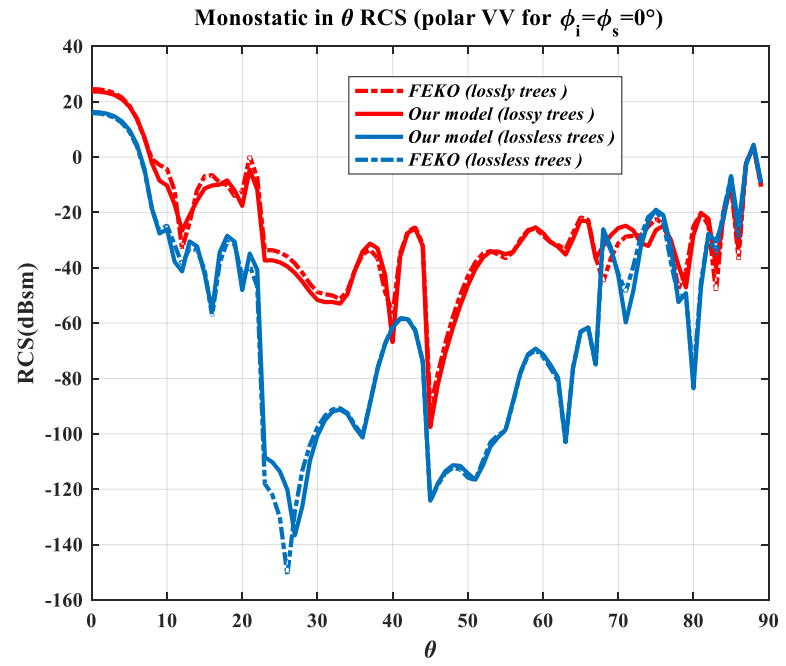

Fig. 6. Comparison of the monostatic RCS obtained with FEKO and our model for the polar $V V$

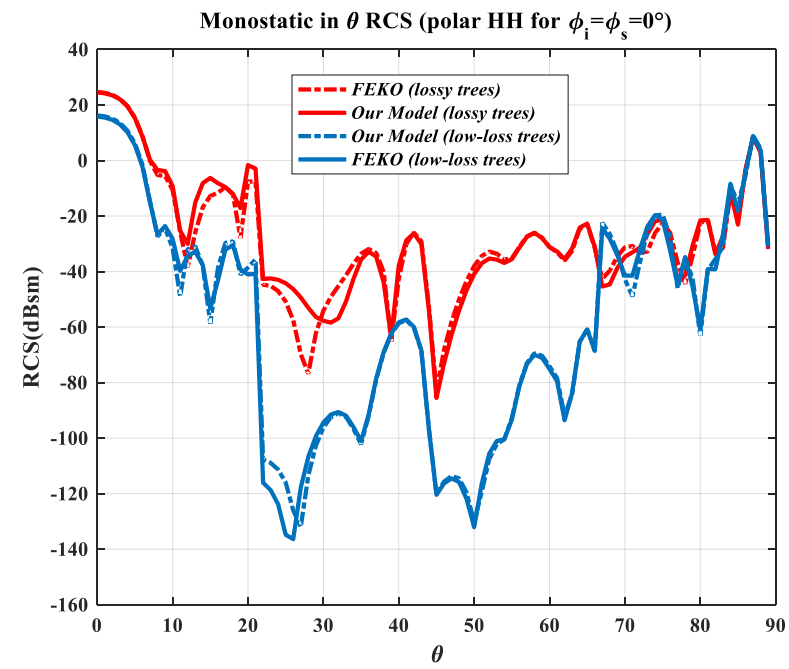

Fig. 7. Comparison of the monostatic RCS obtained with FEKO and our model for the polar $H H$.

\section{Scattering by Metallic targets}

In this section, numerical results for the diffraction of metallic targets placed above a ground plane are presented. The model has been validated at different frequencies (especially in VHF and UHF bands) for several different configurations including metallic structures placed over a lossy ground.

In the considered example (shown in Fig.8), the metallic object is a flat pyramid, $1 \mathrm{~m}$ on the side and $1.5 \mathrm{~m}$ high, placed above a lossy dielectric ground plane.

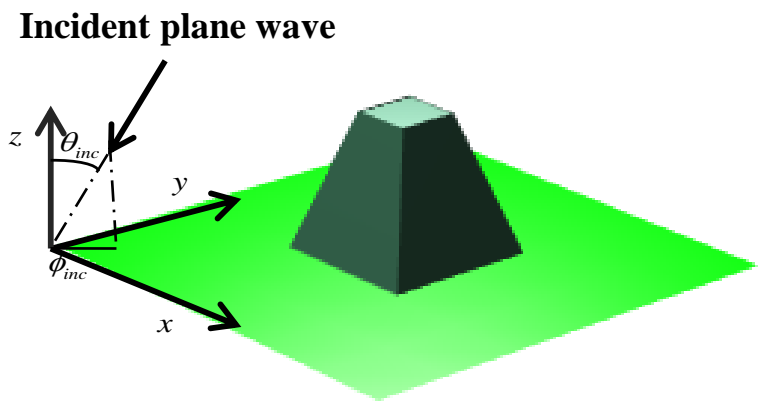

Fig. 8. Geometry of the metallic target

The incident field is a plane wave of frequency $f=700 \mathrm{MHz}$ with incident angle $\theta_{\text {inc }}$ varying from $0^{\circ}$ to $89^{\circ}$ and the azimuth angle $\phi_{i n c}$ is equal to $0^{\circ}$. Two cases of ground are considered, wet and a dry soil. The corresponding material parameters $\left(\varepsilon_{r}\right.$ and $\left.\sigma\right)$ are equal to $\left(20\right.$ and $10^{-2} \mathrm{~S} / \mathrm{m}$ ) and ( 4 and $\left.10^{-3} \mathrm{~S} / \mathrm{m}\right)$, respectively [22].

Figure 9 shows the comparison of the RCS obtained with our model (using image principle with the Fresnel coefficients) and the results derived from the rigorous calculation of the Sommerfeld-integrals (obtained from FEKO). Good agreement is obtained, for both dry and wet soils. 


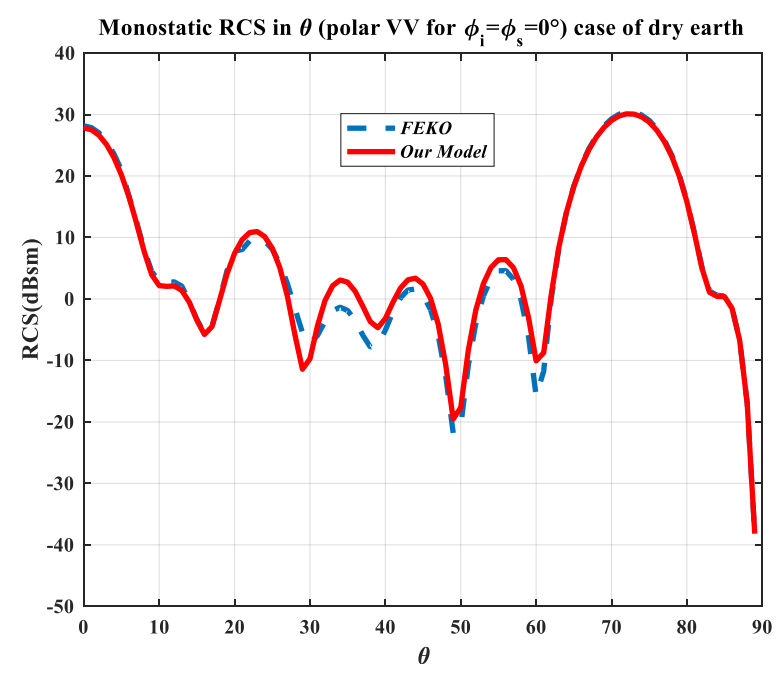

(a)

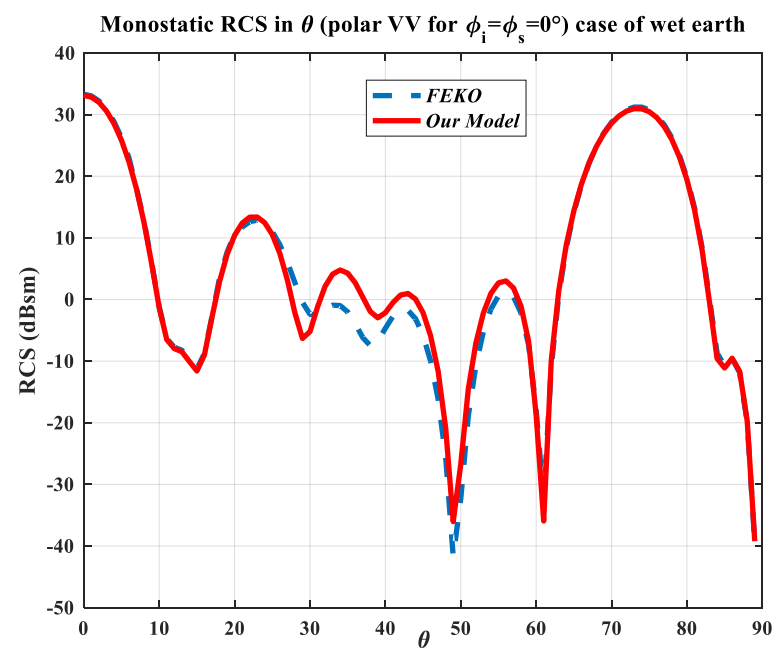

(b)

Fig. 9. Comparison of the variations of the RCS with respect to the incident angle (monostatic case) for the $V V$ polarization (a) dry soil (b) wet soil

\section{Scattering by a Metallic target inside a forest}

The last step of the validation of DEMOS involves the study of a metallic object in presence of the dielectric trees. The considered scenario is composed of a metallic parallelepiped surrounded by two identical trees (see Fig. 10a). The size of the parallelepiped is $(0.2 \mathrm{~m} \times 0.2 \mathrm{~m} \times 1.2 \mathrm{~m})$. The trees are composed of trunks of height $2.1 \mathrm{~m}$ and 4 primary branches. The relative permittivity and conductivity of the trees are $\varepsilon_{r}=4.2$ and $\left.\sigma=0.0167 \mathrm{~S} / \mathrm{m}\right)$. The distance between the cube and the trees is $d=0.25 \mathrm{~m}$. The relative permittivity and conductivity of the ground are $\varepsilon_{r}=5$ and $\sigma=0.034 \mathrm{~S} / \mathrm{m}$, respectively. The incident field is a plane wave propagating along the direction defined by $\left(\theta_{\text {inc }}, \phi_{\text {inc }}\right)=\left(30^{\circ}, 0^{\circ}\right)$ at the frequency of $f=300 \mathrm{MHz}$. The discretization of the scene leads to 7518 unknowns ( 7200 for the volume formulation and 318 for the surface one).

In Fig.10b, a comparison of the amplitude of the diffracted Field (polar HH) obtained with DEMOS and FEKO is presented for an observation point at a distance $R=2000 \mathrm{~m}$.
We note that good agreement is obtained between the two models.

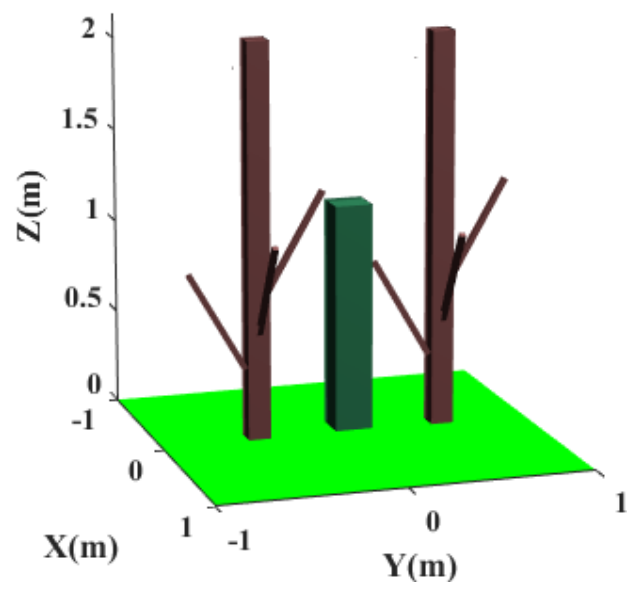

(a)

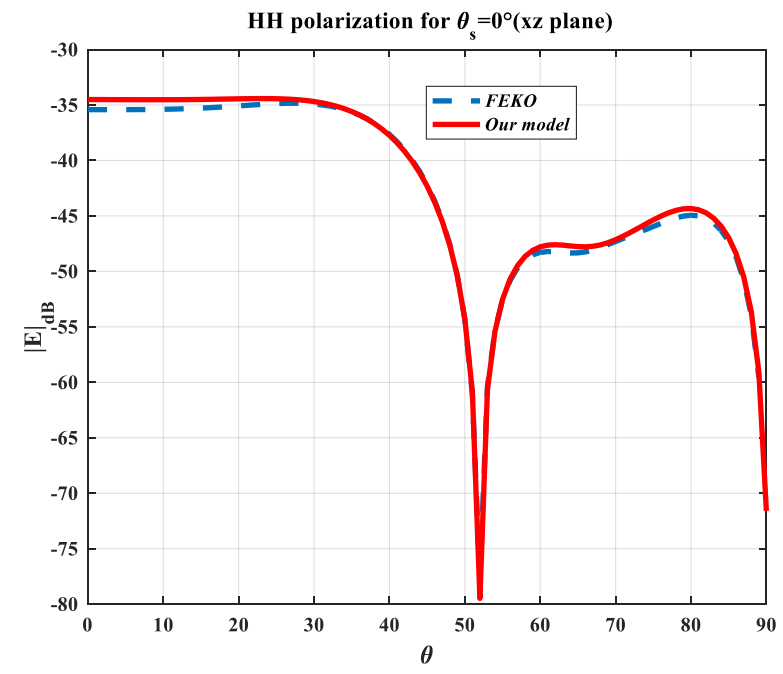

(b)

Fig.10. Comparison of the diffracted field. (a) the considered scene (b) the amplitude of the polar $\mathrm{HH}(\mathrm{dB})$ in the $\phi=0^{\circ}$ cut-plane.

In terms of the computing time, DEMOS is more efficient than FEKO, which uses the traditional MoM. In fact, it took 1 minute and 15 seconds to perform the analysis while FEKO needed around 35 minutes. The execution times were obtained on the same computer (CPU Intel ${ }^{\circledR}$ i7 $4600 \mathrm{U}$ with $16 \mathrm{~Gb}$ RAM).

We conclude, therefore, that DEMOS is well suited for the analysis of complex scenes comprised of metallic objects in the presence of dielectric trees based on the use of hybrid surface-volume formulation. The developed model includes a fast evaluation of the effect of the ground using a simple image method that uses the Fresnel reflection coefficients.

\section{SCATTERING OF A METALLIC TARGET INSIDE A FOREST AREA}

Finally, in this last part of this work, we consider a realistic scenario in order to investigate the effect of a metallic target on the fields scattered by a large forest area (Fig. 11).

The scene is comprised of 52 trees and a metallic tank. Each tree has 8 primary branches and their heights vary between 1.2 $\mathrm{m}$ to $4.5 \mathrm{~m}$. The relative permittivity and conductivity of the 
ground are $\varepsilon_{r}=5$ and $\sigma=0.07 \mathrm{~S} / \mathrm{m}$. The tank is $2.6 \mathrm{~m} \mathrm{high,}$ $3.2 \mathrm{~min}$ length and $1.5 \mathrm{~m}$ in width. The incident field is a plane wave of frequency $f=400 \mathrm{MHz}$. The discretization of the scene leads to 773670 unknowns (762240 for the volume formulation and 11430 for the surface one).

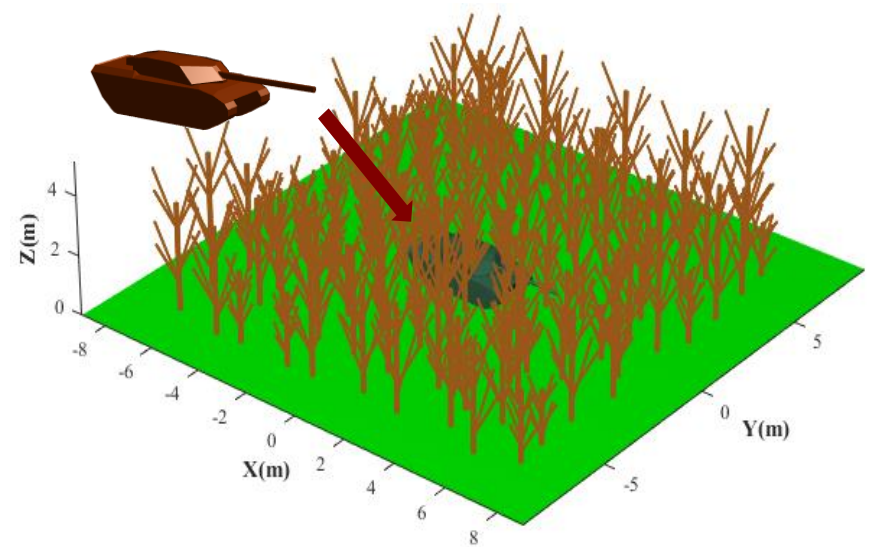

Fig.11. Geometry of the considered scene.

Figures 12 and 13 present the scattered field with the respect to the direction cosines in presence and without the target for the incident angles $\left(\theta_{\text {inc }}, \phi_{\text {inc }}\right)=\left(45^{\circ}, 0^{\circ}\right)$ and $\left(\theta_{i n c}, \phi_{i n c}\right)=\left(45^{\circ}, 120^{\circ}\right)$, respectively.
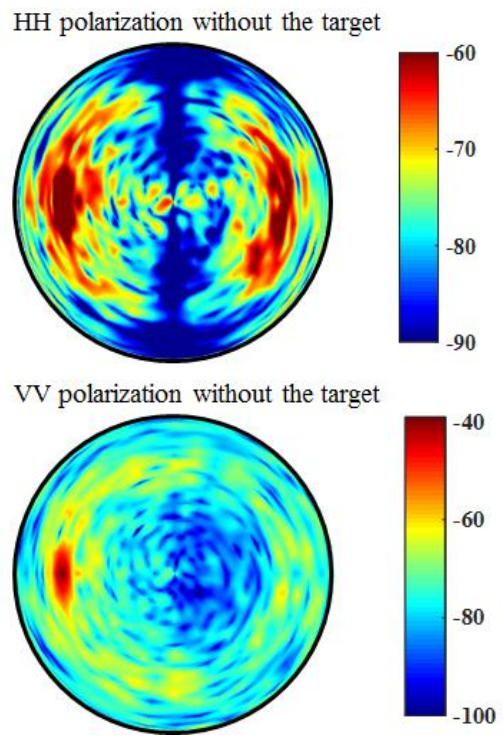

(a)

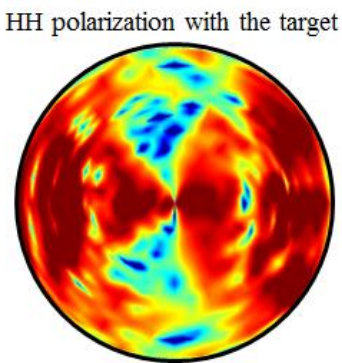

VV polarization with the target

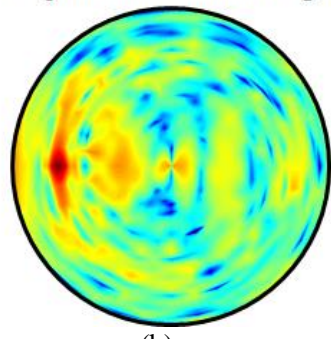

(b)

of vertical trunks and branches with relatively vertical orientations.

It is worthwhile note that the presence of a metallic target (Figs. 12b and 13b) significantly enlarges the aperture of the two main lobes of the pattern. This leads us to the conjecture that the most desirable bi-static angular directions for detecting metallic objects are not in the back- and forwardscattering directions but in the angular ranges that skirt these angles instead.

$\mathrm{HH}$ polarization without the target
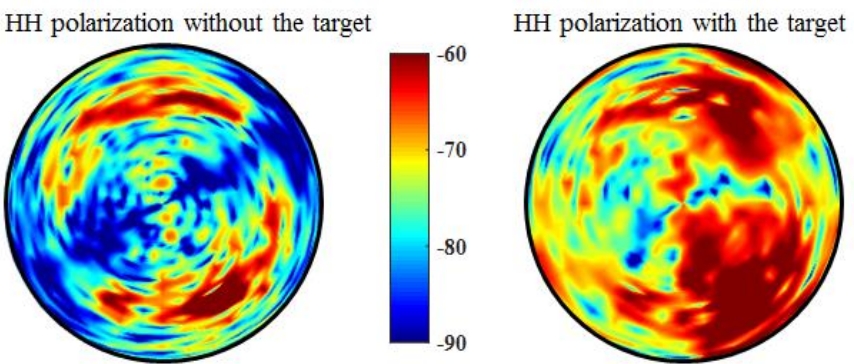

VV polarization without the target

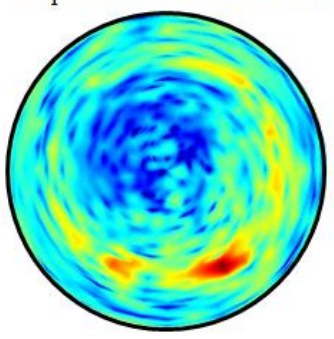

(a)

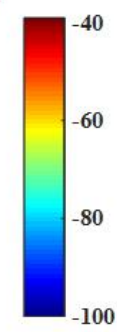

$-100$

VV polarization with the target

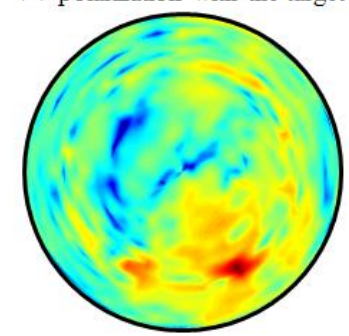

(b)

Fig.13. Influence of the presence of a metallic target in a forest environment on the amplitude of the polar $V V$ and $H H$ for the incident angle $\left(\theta_{i n c}, \phi_{i n c}\right)=\left(45^{\circ}, 120^{\circ}\right)$ : (a) without the target $(\mathrm{dB})$. (b) in presence of the target $(\mathrm{dB})$.

\section{CONCLUSION}

In this paper, an efficient formulation for the analysis of targets placed in a forest environment has been presented. The proposed scheme hybridizes two electromagnetic formulations for dielectric and metallic objects. The model was numerically validated for different test scenarios, obtaining good agreement with the commercial software FEKO. Finally, the effect of metallic targets on the fields diffracted by realistic forest area was investigated from the point of view of detecting these targets, which was the main goal of this study.

\section{ACKNOWLEDGMENT}

This work is supported by the French Agence Nationale de la Recherche (ANR) and the French Direction Générale de l'Armement (DGA) under the Project MOBILE.

\section{REFERENCES}

[1] M. R. Allen, J. M. Jauregui and L. E. Hoff, "FOPEN-SAR detection by direct use of simple scattering physics," Proceedings International Radar Conference, Alexandria, VA, 1995, pp. 152157.

[2] Liao, DaHan, and Traian Dogaru. "Full-Wave Scattering And Imaging Characterization Of Realistic Trees For FOPEN Sensing". IEEE Geoscience and Remote Sensing Letters 13.7 (2016): 957-961. 
[3] Sullivan, Anders. "Advanced Modeling and Simulation of Low Frequency Foliage Penetrating Radar." US Army Research Laboratory, Research Report (1995).

[4] K. Sarabandi, M. Dehmollaian and H. Mosallaei, "Hybrid FDTD and single-scattering theory for simulation of scattering from hard targets camouflaged under forest canopy," in IEEE Transactions on Geoscience and Remote Sensing, vol. 44, no. 8, pp. 2072-2082, Aug. 2006.

[5] M. Dehmollaian and K. Sarabandi, "Electromagnetic Scattering From Foliage Camouflaged Complex Targets," in IEEE Transactions on Geoscience and Remote Sensing, vol. 44, no. 10, pp. 2698-2709, Oct. 2006.

[6] Zhijun Liu, Jiangqi He, Yongjun Xie, A. Sullivan and L. Carin, "Multilevel fast multipole algorithm for general targets on a halfspace interface," in IEEE Transactions on Antennas and Propagation, vol. 50, no. 12, pp. 1838-1849, Dec 2002.

[7] S. M. Rao, T. K. Sarkar, P. Midya and A. R. Djordevic, "Electromagnetic radiation and scattering from finite conducting and dielectric structures: surface/surface formulation," in IEEE Transactions on Antennas and Propagation, vol. 39, no. 7, pp.

[8] C. C. Lu and W. C. Chew, "A coupled surface-volume integral equation approach for the calculation of electromagnetic scattering from composite metallic and material targets," in IEEE Transactions on Antennas and Propagation, vol. 48, no. 12, pp. 1866-1868, Dec 2000.

[9] S. Bellez, C. Dahon and H. Roussel, "Analysis of the Main Scattering Mechanisms in Forested Areas: An Integral Representation Approach for Monostatic Radar Configurations," in IEEE Transactions on Geoscience and Remote Sensing, vol. 47, no. 12, pp. 4153-4166, Dec. 2009.

[10] I. Fenni, H. Roussel, M. Darces and R. Mittra, "Fast Analysis of Large 3-D Dielectric Scattering Problems Arising in Remote Sensing of Forest Areas Using the CBFM," in IEEE Transactions on Antennas and Propagation, vol. 62, no. 8, pp. 4282-4291, Aug. 2014.

[11] R. Mittra, K. Du, "Characteristic basis function method for iteration-free solution of large method of moments problems", Progr. Electromagn. Res. B, vol. 6, pp. 307-336, Apr. 2008.
[12] S. Rao, D. Wilton and A. Glisson, "Electromagnetic scattering by surfaces of arbitrary shape," in IEEE Transactions on Antennas and Propagation, vol. 30, no. 3, pp. 409-418, May 1982.

[13] Michalski, Krzysztof A. "The mixed-potential electric field integral equation for objects in layered media." Archiv für elektronik und übertragungstechnik 39 (1985): 317-322.

[14] K. A. Michalski and D. Zheng, "Electromagnetic scattering and radiation by surfaces of arbitrary shape in layered media. I. Theory," in IEEE Transactions on Antennas and Propagation, vol. 38, no. 3, pp. 335-344, Mar 1990.

[15] K. A. Michalski and D. Zheng, "Electromagnetic scattering and radiation by surfaces of arbitrary shape in layered media. II. Implementation and results for contiguous half-spaces," in IEEE Transactions on Antennas and Propagation, vol. 38, no. 3, pp. 345352, Mar 1990.

[16] H. Nguyen, H. Roussel and W. Tabbara, "A coherent model of forest scattering and SAR imaging in the VHF and UHF-band," in IEEE Transactions on Geoscience and Remote Sensing, vol. 44, no. 4, pp. 838-848, April 2006.

[17] M. I. Aksun, "A robust approach for the derivation of closed-form Green's functions," in IEEE Transactions on Microwave Theory and Techniques, vol. 44, no. 5, pp. 651-658, May 1996.

[18] A. Alparslan, M. I. Aksun and K. A. Michalski, "Closed-Form Green's Functions in Planar Layered Media for All Ranges and Materials," in IEEE Transactions on Microwave Theory and Techniques, vol. 58, no. 3, pp. 602-613, March 2010.

[19] A. Shoory, R. Moini and S. H. H. Sadeghi, "Direct use of discrete complex image method for evaluating electric field expressions in a lossy half space," in IET Microwaves, Antennas \& Propagation, vol. 4, no. 2, pp. 258-268, Feb. 2010.

[20] Diestel, W. "Arbaro- tree generation for povray." (2003).[online]. Available: http://arbaro.sourceforge.net.

[21] J. E. Hipp, "'Soil electromagnetic parameters as a function of frequency soil density and soil moisture"", Proc. IEEE, vol. 62, pp. 98-103, 1974

[22] D. Liao and T. Dogaru, "Large-scale, full-wave scattering phenomenology characterization of realistic trees," 2015 IEEE International Symposium on Antennas and Propagation \& USNC/URSI National Radio Science. 